\title{
TITLE:
}

\section{CesA protein is included in the terminal complex of Acetobacter}

$\operatorname{AUTHOR}(S)$ :

Sun, Shi-jing; Imai, Tomoya; Sugiyama, Junji;

Kimura, Satoshi

\section{CITATION:}

Sun, Shi-jing ... [et al]. CesA protein is included in the terminal complex of Acetobacter. Cellulose 2017, 24(5): 2017-2027

\section{ISSUE DATE:}

2017-05

URL:

http://hdl.handle.net/2433/230960

\section{RIGHT:}

This is a post-peer-review, pre-copyedit version of an article published in 'Cellulose'. The final authenticated version is available online at: https://doi.org/10.1007/s10570-017-1237-6.; The full-text file will be made open to the public on 1 May 2017 in accordance with publisher's 'Terms and Conditions for Self-Archiving'.; この論文は出版社版でありません 。引用の際には出版社版をご確認ご利用ください。; This is not the published version. Please cite only the published version. 
2 CesA protein is included in the terminal complex of

3 Acetobacter

5 Shi-jing Sun, Tomoya Imai, Junji Sugiyama, Satoshi Kimura ${ }^{\dagger}$

7 Research Institute for Sustainable Humanosphere (RISH), Kyoto University, Uji, 8 Kyoto 611-0011, Japan

$9{ }^{\dagger}$ Graduate School of Agricultural and Life Science, the University of Tokyo, 10 Yayoi 1-1-1, Bunkyo-ku, Tokyo 113-0011 Japan

12 To whom correspondence may be addressed: Tomoya Imai, RISH, Kyoto

13 University, Uji, Kyoto 611-0011, Japan.

14 Tel: +81-774-38-3631

15 E-mail: timai@ rish.kyoto-u.ac.jp

17 Running title: CesA in bacterial terminal complex

19 Abstract

20 Cellulose is a major biopolymer on the earth that is derived from cellulose synthase in the cell

21 membrane of living organisms. Cellulose synthase is a hetero-subunit complex composed of

22 several different protein subunits, and is visualized as a supermolecular complex called a "terminal

23 complex" by electron microscopy. Such supermolecular organization of an enzyme complex is

24 believed to be important for the fiber formation or crystallization of cellulose microfibrils in

25 cellulose biosynthesis. In the case of the cellulose-producing bacterium Acetobacter, it is

26 hypothesized that the enzyme complex includes at least six subunits given its genetic constitution.

27 However, to date, only three of these molecules have been experimentally confirmed as the

28 subunits included in the cellulose synthase complex: CesB, CesD, and ccp2. In this study, we used

29 fluorescence immuno-microscopy to show that CesA protein, the catalytic subunit, is included in

30 the terminal complex of Acetobacter. Furthermore we discuss the obtained microscopic data for

31 improving our understanding of the molecular organization of the bacterial cellulose synthase

32 complex. 
34 Keywords: CesA; cellulose synthase complex; terminal complex; immunolabeling 35

36 Abbreviations: PFA, paraformaldehyde; EDTA, ethylenediamine tetra-acetic acid; SDS, sodium

37 dodecyl sulfate; PAGE, poly-acrylamide gel electrophoresis; PVDF, poly-vinylidene difluoride;

38 RT, room temperature; BSA, bovine serum albumin

39

40 
INTRODUCTION

Cellulose is one of the major biopolymers on Earth. Despite its mass

43 production on Earth by plants and other living organisms, destructive

44 accumulation of cellulose on Earth has never been identified, although this has

45 been observed for some synthetic plastics. This indicates that the cycle of

46 synthesis and degradation shows a good balance for maintaining the cellulose

47 content on Earth, which is actually a striking feature of cellulose, suggesting it as

48 a promising material for sustainable human life.

All of the cellulose on Earth is produced by living organisms, and

50 originates from the cellulose synthase complex (CSC) in living cells. CSC is a

51 hetero-subunit complex in the cell membrane (Somerville 2006). Electron

52 microscopy with the freeze-fracture technique has been used to visualize CSC as a

53 terminal complex (TC), which is a characteristic array of particles found on the

54 cell membrane at the terminal of cellulose microfibrils (Kimura et al. 1999,

55 Kimura et al. 2001). Since the 1970s, TCs have been found in many of the

56 cellulose-producing organisms (a tunicate, algae, plants, and a bacterium),

57 although the arrangement of the TC particles showed a variety of patterns (Itoh et

58 al. 2007): linear type (for a bacterium, tunicate, and algae producing a giant

59 microfibril) and rosette-type (for higher plants and an alga of the order

60 Zygnematale). Regardless of the specific pattern, such a regular array of a

61 cellulose-synthesizing enzyme is considered to be important for cellulose

62 microfibril formation by assembling many cellulose chains into a cellulose

63 microfibril.

To date, several studies have identified the molecules included in the CSC

65 based on biochemical and molecular/cell biological analyses. For Acetobacter 
66 (recently renamed for some strains as Gluconacetobacter, Komagataeibacter, and

67 so on), a popular model for studying cellulose biosynthesis, six subunits are

68 proposed to be included in the CSC given the constitution of the genes related to

69 cellulose synthesis (McNamara et al. 2015): GH-8 (also known as carboxymethyl

70 cellulase (CMC)) (Standal et al. 1994), cellulose complementing factor (ccp)

71 (Standal et al. 1994), CesA, CesB, CesC, and CesD (Saxena et al. 1994, Wong et

72 al. 1990). Among these, CesA is the catalytic subunit harboring the

73 glycosyltransferase domain of the GT-2 family in the cytosolic part (Morgan et al.

74 2013), and CesA and CesB are the minimally required subunits for cellulose-

75 synthesizing activity (Omadjela et al. 2013, Saxena et al. 1994, Wong et al. 1990).

76 CesD is considered to control the crystallization process of cellulose microfibrils

77 (Hu et al. 2010, Saxena et al. 1994), and four chains are found inside the ring

78 structure formed by the octamer of CesD protein (Hu et al. 2010). The functions

79 of the other subunits have not yet been clarified despite their clear relevance to

80 cellulose-synthesizing activity, as experimentally reported for GH-8 (Kawano et

81 al. 2002, Kawano et al. 2008, Nakai et al. 2013), ccp (Sunagawa et al. 2013),

82 CesC (Saxena et al. 1994), and CesD (Hu et al. 2010, Saxena et al. 1994,

83 Sunagawa et al. 2013).

The SDS-freeze replica labeling (SDS-FRL) method (Fujimoto 1995), an

85 immuno-labeling technique combined with the freeze-replica technique, is a direct

86 method to localize a specific protein in the TCs, and its application has shown that

87 CesB protein is found in the linear TC of Acetobacter (Kimura et al. 2001). In

88 addition, fluorescence microscopy could also be used to successfully visualize the

89 linear localization of GFP-fused CesD and ccp protein in the cells of Acetobacter

90 (Sunagawa et al. 2013), which confirmed that these two proteins are also included 
91 in the linear TC of Acetobacter. These microscopic studies showed that CesB,

92 CesD, and ccp proteins form a part of the TC or CSC of Acetobacter. However,

93 no report has provided concrete evidence that CesA protein is included in the

94 linear TC of Acetobacter, although this is the widely accepted hypothesis given

95 that CesA is the core subunit of the CSC. In the present study, CesA protein was

96 successfully visualized as a linear array in the cell, and experimental evidence was

97 obtained to show that CesA protein is included in the TC of Acetobacter.

99 MATERIALS AND METHODS

100 Chemicals

101 Peptone and yeast extract for the culture medium were purchased from

102 Becton, Dickinson and Company Inc. (USA). Paraformaldehyde (PFA) for cell

103 fixation was paraformaldehyde EM from TAAB Inc. (UK). Poly-L-lysine

104 solution, lysozyme and BSA were purchased from Sigma-Aldrich Inc. The other

105 chemicals used in this study were purchased from Wako Pure Chemicals Inc.

106 (Japan) unless described.

107 Cell culture

108 Three different strains of Acetobacter were used in this study:

109 ATCC53264, ATCC53524, and JCM9730. For convenience, the former name

110 Acetobacter is used for these strains herein, although these are actually considered

111 to be different species based on the current taxonomy (Gluconacetobacter xylinus

112 for ATCC53264, Komagataeibacter xylinus for ATCC53524, and

113 Komagataeibacter sucrofermentans for JCM9730). The two ATCC strains were

114 provided by the American Type Culture Collection and the last strain was

115 obtained from the Japan Collection Microorganisms at BRC-RIKEN, Japan. Each 
116 strain was grown in Schramm-Hestrin medium (Schramm and Hestrin 1954) at

$11730^{\circ} \mathrm{C}$ in a static condition for 3 to 5 days, until a sufficient amount of cellulose

118 was produced. The cells were detached from the cellulose pellicle by shaking the

119 culture medium and pressing the pellicle with a spatula, and then filtered by 37 - or

$120 \quad 50-\mu \mathrm{m}$ pore-sized nylon mesh. The filtrated cells were collected by centrifugation

$121(2000 \times g$ for $10 \mathrm{~min}$ at RT).

122 Antibody evaluation by western blot analysis

123 Western blot analysis was performed to evaluate whether the primary

124 antibody has cross-reactivity with the proteins in the strains ATCC53264 and

125 JCM9730, as well as strain ATCC53524 for which cross-reactivity has already

126 been shown (Hashimoto et al. 2011). The primary antibodies used in this study

127 were the same as those used in our previous studies (Hashimoto et al. 2011, Imai

128 et al. 2014, Sun et al. 2016). In brief, each antibody is a polyclonal antibody

129 against the synthetic peptide corresponding to a part of CesA (carboxyl terminal),

130 CesB (a loop in the CBD2 domain), CesC (the part between the last six-TPR

131 repeat and the carboxyl terminal region), and the CesD subunit (the loop between

132 the $\beta 3$ and $\beta 4$ strands). The antigen peptide sequence for each of the proteins was

133 designed from the sequence of the strain BPR2001 (Nakai et al. 1998) or

134 JCM9730 (GenBank: AB010645) as reported in our previous study (Hashimoto et

135 al. 2011). As shown in Table 1, high sequence similarity was found for each of the

136 proteins between this strain and ATCC53264 or 1306-03 (GenBank: AAA21884 -

137 21887), and probably ATCC53524 or 1306-21, which is a derivative strain of

138 ATCC53264 (Wong et al. 1990). 
140 Table 1. Amino acid sequences of the peptide antigens for the antibodies used in this study

141 (JCM9730), together with the sequence of the corresponding part for ATCC53264. The non-

142 identical residues are indicated with shadowing.

Protein and strain

Amino acid sequence

\begin{tabular}{|c|c|c|c|c|c|c|c|c|c|c|c|c|c|c|c|c|}
\hline \multirow{2}{*}{ CesA } & JCM9730 (antigen) & $S$ & $\mathrm{G}$ & Q & $\mathrm{T}$ & $Q$ & $E$ & $\mathrm{G}$ & K & I & $S$ & $\mathrm{R}$ & A & $A$ & $S$ & \\
\hline & ATCC53264 & $\mathrm{S}$ & G & Q & $\mathrm{T}$ & Q & $E$ & G & K & I & $S$ & $\mathrm{R}$ & A & $A$ & S & \\
\hline \multirow{2}{*}{ CesB } & JCM9730 (antigen) & $\mathrm{S}$ & $P$ & D & L & Y & $\mathrm{T}$ & W & $\mathrm{R}$ & D & $\mathrm{R}$ & $P$ & $\mathrm{~N}$ & K & & \\
\hline & ATCC53264 & $\mathrm{S}$ & $P$ & D & L & Y & $\mathrm{T}$ & W & $\mathrm{R}$ & D & $\mathrm{R}$ & $P$ & $Y$ & $\mathrm{~K}$ & & \\
\hline \multirow{2}{*}{$\mathrm{CesC}$} & JCM9730 (antigen) & $P$ & S & I & D & $\mathrm{G}$ & $\mathrm{G}$ & L & $G$ & $\mathrm{~F}$ & $\mathrm{R}$ & $S$ & $\mathrm{R}$ & $S$ & G & $E$ \\
\hline & ATCC53264 & $\mathrm{P}$ & $S$ & I & D & $\mathrm{G}$ & $\mathrm{G}$ & L & G & $F$ & $\mathrm{R}$ & $S$ & $\mathrm{R}$ & $S$ & G & $E$ \\
\hline \multirow{2}{*}{ CesD } & JCM9730 (antigen) & $\mathrm{T}$ & $\mathrm{R}$ & D & I & D & A & $E$ & D & L & $\mathrm{N}$ & $S$ & & & & \\
\hline & ATCC53264 & $\mathrm{T}$ & $\mathrm{R}$ & D & I & D & A & $E$ & D & $\mathrm{L}$ & $\mathrm{N}$ & $S$ & & & & \\
\hline
\end{tabular}

144 The centrifuged cells described above were resuspended in a buffer of 10

$145 \mathrm{mM}$ Tris- $\mathrm{HCl}(\mathrm{pH} 8.0), 5$ mM EDTA, $0.02 \% \mathrm{NaN}_{3}, 50 \mu \mathrm{g} / \mathrm{mL}$ chloramphenicol.

146 Then, the cell suspension was mixed with the SDS-PAGE sample buffer. After

147 incubating at $4{ }^{\circ} \mathrm{C}$ for overnight, the sample was analyzed with a precast gel with a

148 gradient of 5-20\% acrylamide (SuperSep Ace, Wako Pure Chemicals Industries

149 Ltd., Japan). The band pattern was transferred from the gel to a PVDF membrane

150 (Immobilon-P, Millipore Inc.), and then the membrane was incubated with each of

151 the primary antibodies against $\mathrm{Ces} A, \mathrm{CesB}, \mathrm{CesC}$, and CesD protein. Finally, the

152 protein band was visualized on the PVDF membrane by a chemical luminescence 
153 method with ECL select (GE Healthcare Inc.) and recorded by a CCD camera

154 (EZ-capture, ATTO Inc., Japan).

The centrifuged cells were resuspended in CBS (citrate buffered saline:

$15750 \mathrm{mM}$ sodium citrate buffer ( $\mathrm{pH} 5.0), 136 \mathrm{mM} \mathrm{NaCl}, 2.7 \mathrm{mM} \mathrm{KCl})$ and then

158 incubated in 2\% PFA in CBS at $4{ }^{\circ} \mathrm{C}$ overnight to chemically fix the cells. Then,

159 the gently centrifuged cells $(1000 \times g$ for $10 \mathrm{~min}$ at RT) were resuspended in PBS

160 (phosphate buffered saline: $10 \mathrm{mM}$ phosphate buffer (pH7.4), $136 \mathrm{mM} \mathrm{NaCl}, 2.7$

$161 \mathrm{mM} \mathrm{KCl}$ ) with $0.1 \mathrm{M}$ glycine for quenching the PFA. The cell suspension was

162 dropped on the glass coverslip, which was made to be hydrophilic in advance by

163 dipping in $1 \mathrm{mg} / \mathrm{mL}$ poly-L-lysine solution at RT for $30 \mathrm{~min}$. The coverslip

164 carrying the cells was processed with the following procedures of lysozyme

165 treatment and permeabilizing treatment, prior to the antibody treatment.

166 The cells on the coverslip were treated with $1 \mathrm{mg} / \mathrm{mL}$ lysozyme in TE

167 buffer (100 mM Tris- $\mathrm{HCl}(\mathrm{pH}$ 6.7), $5 \mathrm{mM} \mathrm{EDTA})$ at $37^{\circ} \mathrm{C}$ for $1 \mathrm{~h}$. After four

168 repeated washes with PBS, the cells were permeabilized with 1\% IGEPAL CA-

169630 (MP Biomedicals LLC; equivalent to a detergent Nonidet P-40) in PBS at

$17030^{\circ} \mathrm{C}$ for $30 \mathrm{~min}$. The cells were then washed four times with PBS for 5 min each

171 time. Some of these treatments were skipped to explore the subunit localization in

172 the cell.

173 Immunolabeling of the cells

174 Prior to the antibody treatment, the cells on the coverslip were incubated in

$1751 \%$ BSA and 1\% Blocking Reagent (Roche Inc.) in PBS at RT for $1 \mathrm{~h}$ for

176 blocking. Then, the cells were treated with the primary antibody solution, which

177 was diluted 500-fold in the blocking buffer, at $4^{\circ} \mathrm{C}$ for overnight with gentle 
178 shaking. After four washes with PBS every $5 \mathrm{~min}$, the cells were treated with 5

$179 \mu \mathrm{g} / \mathrm{mL}$ of the fluorophore-conjugated anti-rabbit IgG (Alexa fluor 488, Thermo

180 Fisher Scientific Inc.) in the blocking buffer at RT for $2 \mathrm{~h}$ in the dark. The cells on

181 the coverslip were washed with PBS four times every 5 min. The coverslip was

182 taken out to wash the side without the cells in pure water, and then placed on the

183 slide glass to seal the cells in the anti-fading reagent (SlowFade, Invitrogen Inc.).

184 Control experiments were done for the fixed Acetobacter cells by using either no

185 primary antibody or fluorophore-conjugated anti-mouse IgG (Alexa fluor 488,

186 Thermo Fisher Scientific Inc.) for the secondary antibody.

187 The cells on the slide glass were observed on an IX71 microscope

188 (Olympus Inc., Japan) with an oil immersion lens. Epi-fluorescence mode with a

189 mercury lamp and the filter set FITC-2024B (Semrock Inc., USA) was used for

190 recording the fluorescence image by a CCD camera (DP73, Olympus Inc., Japan).

191 The same region of interest was also recorded with the phase-contrast mode for

192 subsequent merging with the fluorescence image.

193 SDS-freeze replica labeling

194 The cells collected from the pellicle as described above were quickly

195 frozen on the gold sample career by dipping into liquid ethane at $-175^{\circ} \mathrm{C}$ with a

196 Leichert KF-80 system (Leica Inc.). The freeze-fracture replica of these cells

197 without chemical fixation was prepared using a BAF-400D system (Balzers Inc.).

198 The fracture was performed at $-113^{\circ} \mathrm{C}$, and then platinum/carbon was evaporated

199 on the fractured surface at an angle of $45^{\circ}$ followed by rotary carbon coating to

200 support the platinum replica. The prepared replica was treated in the lysozyme

201 solution ( $1 \mathrm{mg} / \mathrm{mL}$ lysozyme in $25 \mathrm{mM}$ Tris- $\mathrm{HCl}(\mathrm{pH}$ 8.0), $10 \mathrm{mM}$ EDTA) for $4 \mathrm{~h}$

202 at RT, and subsequently in the lysis solution (2.5\% SDS, $10 \mathrm{mM}$ Tris- $\mathrm{HCl}(\mathrm{pH}$ 
203 8.0)) for $2 \mathrm{~h}$ at RT. After washing three times with PBS, the replica was then

204 incubated in the blocking solution (1\% BSA in PBS) for $30 \mathrm{~min}$ at RT, and then

205 treated with the primary antibody diluted in the blocking buffer overnight at $4^{\circ} \mathrm{C}$.

206 The replica was then washed in PBS with 0.05\% Tween-20 (PBST) and treated

207 with the secondary antibody (anti-rabbit IgG conjugated with 15-nm colloidal

208 gold, British BioCell International, UK) for $1.5 \mathrm{~h}$ at RT. Finally, the replica was

209 treated with $0.5 \%$ glutaraldehyde in PBS for 15 min at RT, and then transferred on

210 the carbon-coated copper grid after washing with water.

211 The replica on the grid was observed by a JEM-2000EXII (Jeol Inc.,

212 Japan) electron microscope and the images were recorded with photo-emulsion

213 (FG film, FujiFilm Inc., Japan), which was developed by Korectol (FujiFilm Inc.,

214 Japan) for 4 min at $20^{\circ} \mathrm{C}$.

215

216

217

218

219 RESULTS

220 CesA is present in the linear array in the bacterial cells

222 study are shown in Figure 1. These antibodies basically showed cross-reactivity to

223 the proteins extracted from Acetobacter in the three different strains used in this

224 study (ATCC 53524, ATCC53264, and JCM9730). We then used these antibodies

225 for immunolabeling fluorescence microscopy, as shown in Figure 2. 
A

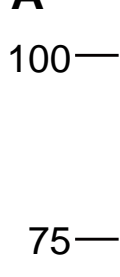

(kDa)

C

$150-$

$100-$

227

228

229

230

231

232

233

234

235

236

237

238

239

240

241 in this study. This clearly indicates that CesA and CesD proteins are the subunits

242 included in the linear TC of Acetobacter. Furthermore the linear signal was

243 sometimes found at the lateral edge of the cell on the micrograph, indicating that

244 the labeled protein is not on the inside but rather at the boundary of the

245

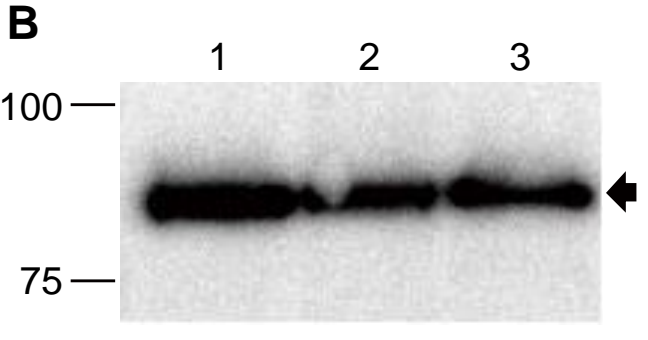

$(\mathrm{kDa})$

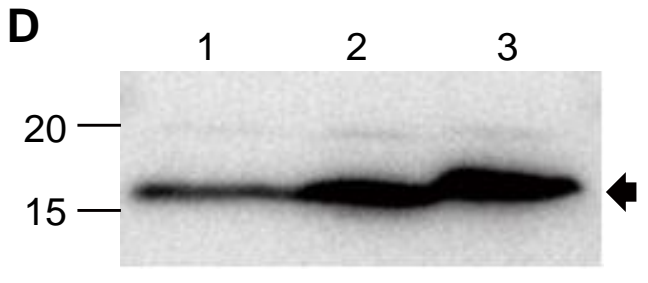

$(\mathrm{kDa})$
Figure 1.

Western blot analysis with SDS-PAGE for the whole cell sample of Acetobacter cells. A, B, C, and $\mathrm{D}$ show the results with the antibody against CesA, CesB, CesC, and CesD protein, respectively. The sample of ATCC53524, ATCC53264, and JCM9730 was loaded into the lane 1, 2 , and 3, respectively. Roughly the same number of cells, measured by the optical density at 600 $\mathrm{nm}$, were loaded. The arrow indicates the band of interest.

treatment, and detergent treatment) as well as the antibody treatment, we could successfully label CesA and CesD proteins as a linear array in the cell (Figure 2A) whereas no labeling was found in negative controls (Figure 2B). Such a linear labeling pattern was not found for the immunolabeling of CesB and CesC. The linear labeling pattern of CesA and CesD was observed for all of the strains used Acetobacter cell. Therefore, the linear immunolabeling pattern shown in Figure 
$2462 \mathrm{~A}$ provides the experimental evidence that CesA and CesD are included in the

247 linear TC on the cell membrane, the bacterial CSC.

248

A
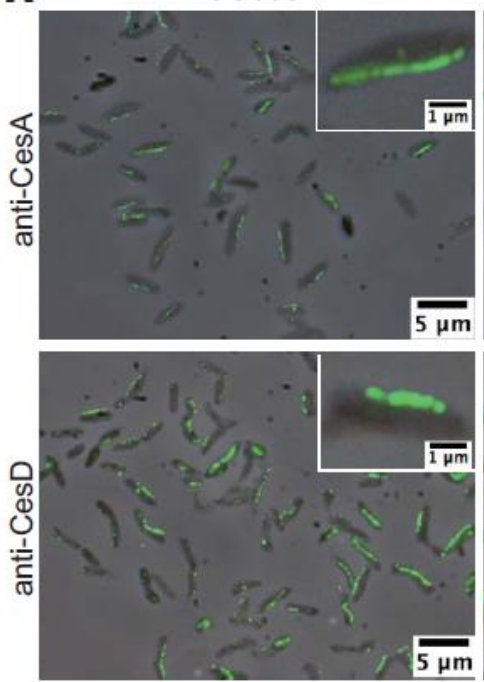

$\overline{5 \mu \mathrm{m}}$

B

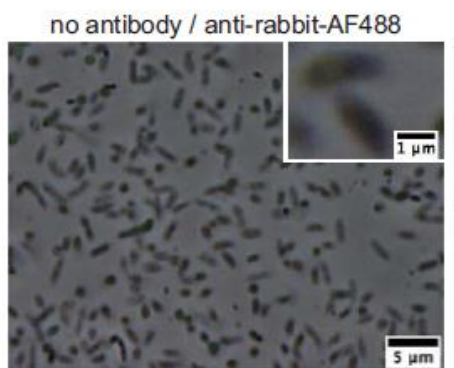

ATCC53264
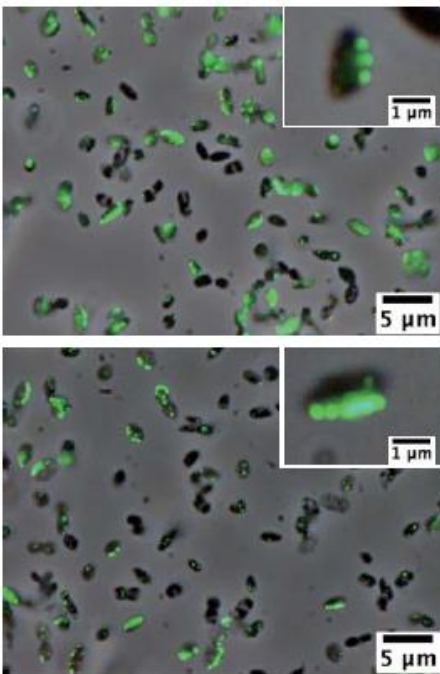
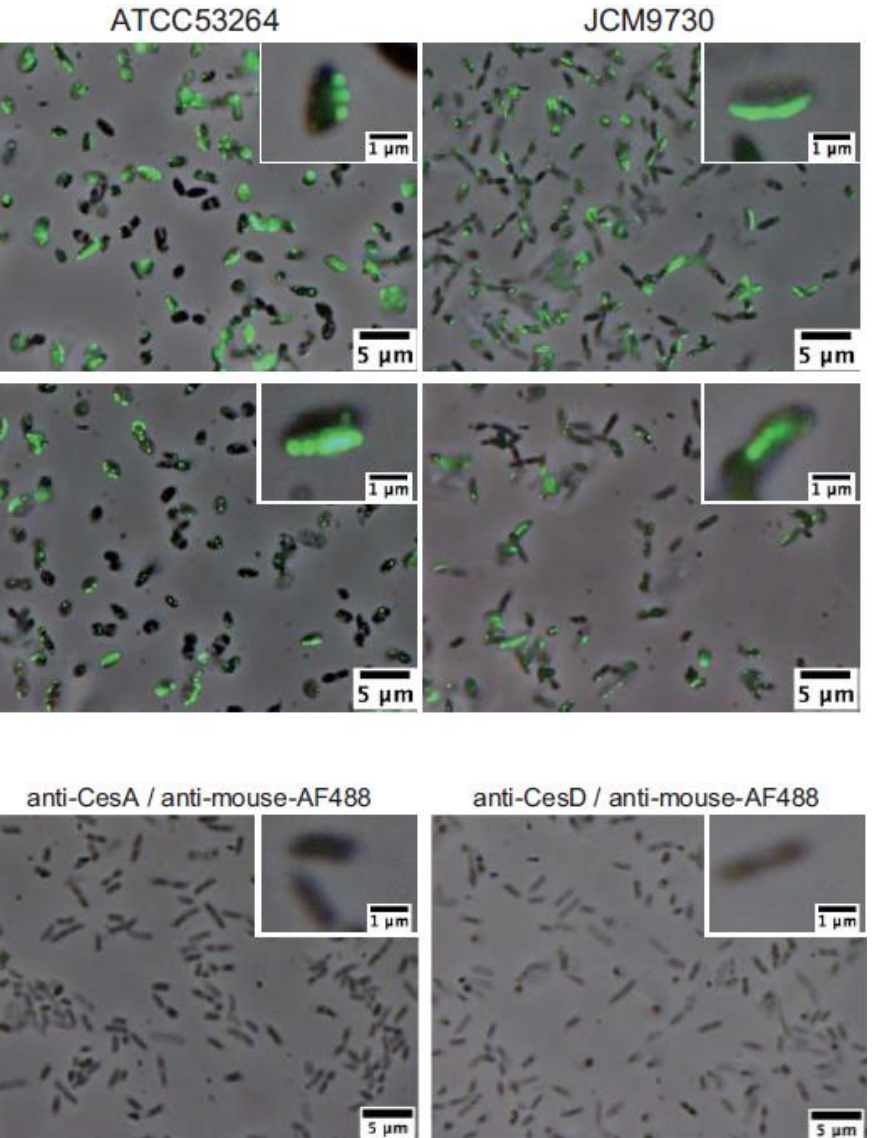

anti-CesD / anti-mouse-AF488

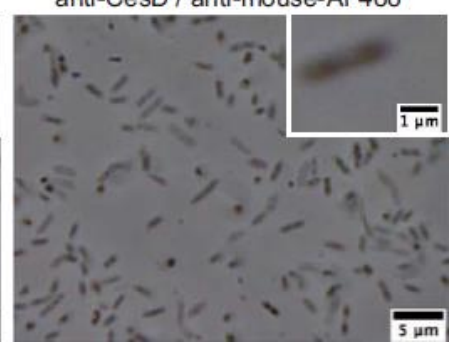

Figure 2.

251 Fluorescence micrographs of Acetobacter cells with immunolabeling by the antibodies against

252 CesA and CesD proteins. The phase-contrast images and the epi-fluorescence image are merged.

253 (A) Three different strains (ATCC53524, ATCC53264, and JCM9730) were labeled using the

254 identical protocol with a correct choice of the antibodies. The inset shows the image at a higher

255 magnification. (B) Control experiments with a strain ATCC53524. Combination of the

256 primary/secondary antibodies was used as indicated. Almost no labeling was found in neither

257 conditions.

259 Change of the immunolabeling efficiency for CesA and CesD protein.

261 adequate procedure prior to labeling. In the case of the bacterial cell, lysozyme 
262 treatment is commonly used for disintegrating the peptidoglycan layer beneath the

263 outer membrane, and detergent treatment is used for permeabilizing the outer and

264 inner membrane. Therefore, in principle, the protein exposed to the outside of the

265 cell will be labeled without any pretreatment. We then surveyed the change in the

266 immunolabeling efficiency depending on the pretreatment applied to explore the

267 location of CesA and CesD proteins, which were successfully immunolabeled in

268 this study. The strain ATCC53524 was used for this purpose given the fact that

269 this strain showed the highest immunolabeling efficiency.

270 We tested five different pretreatments, in addition to the optimized

271 condition shown above (Figures 3A and 4A): (i) no treatments, (ii) EDTA

272 treatment, (iii) detergent treatment, (iv) EDTA treatment followed by detergent

273 treatment, and (v) lysozyme treatment (Figures 3 and 4, and summarized in Table

274 2). First, the cells with no pretreatment showed almost no immunolabeling for

275 neither CesA nor CesD (Figures 3B and 4B), indicating that CesA and CesD are

276 not exposed to the outside of the cell. Notably, EDTA treatment alone allowed for

277 the immunolabeling of CesD but not CesA (Figures 3C and 4C). Given the

278 relatively mild disturbance of the outer membrane only by depletion of divalent

279 cations with EDTA, CesD is probably located in the periplasmic space and was

280 immunolabeled due to access of the antibody. By contrast, CesA protein is a

281 transmembrane protein, with its carboxyl terminal (the epitope of the antibody

282 used in this study) facing to the cytoplasm. Therefore, it is reasonable that CesA

283 was not immunolabeled for cells whose outer membrane is mildly disturbed by

284 EDTA alone, which is not harsh enough to allow for cell lysis. 

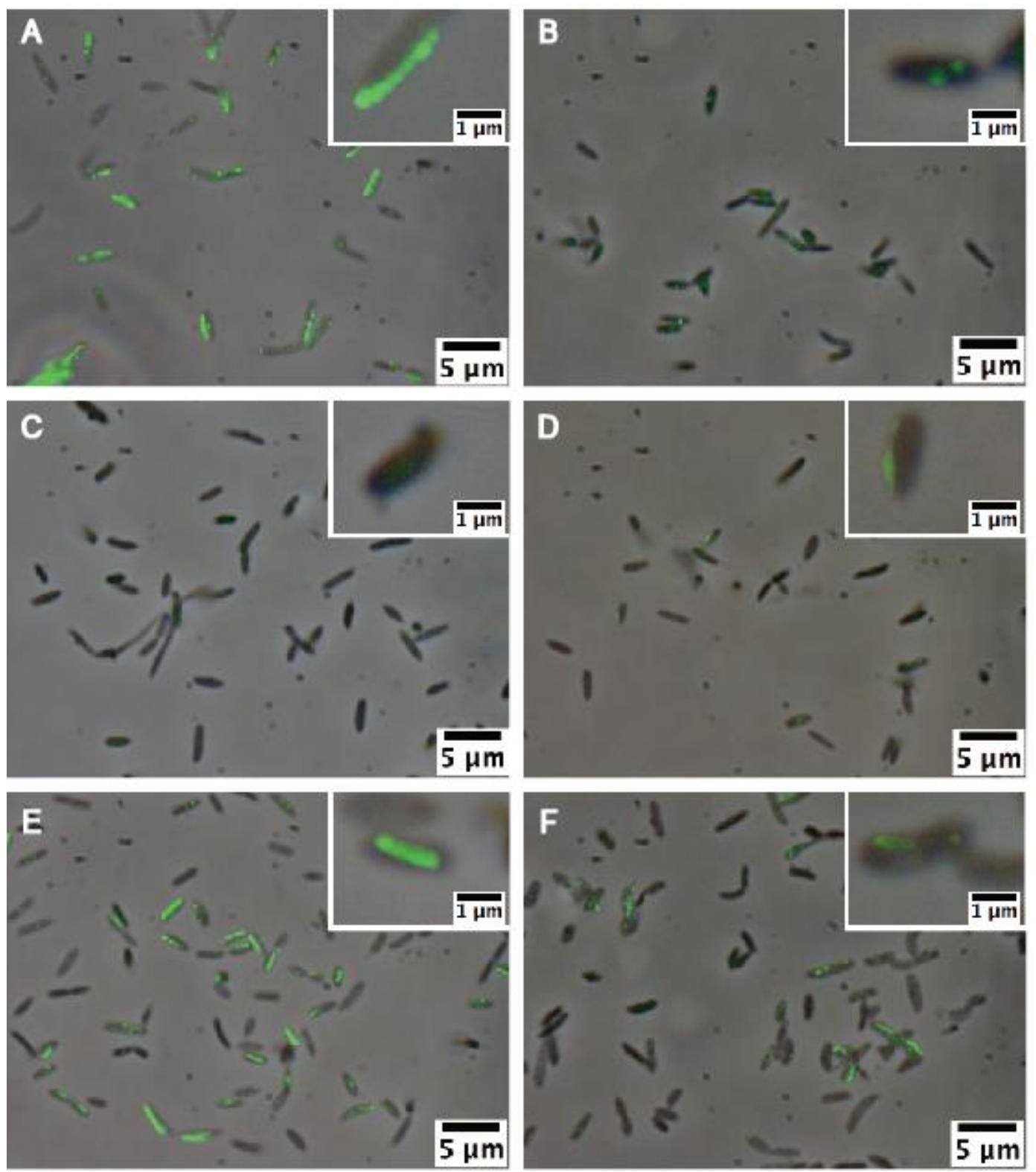

Figure 3.

288 Fluorescence micrographs with immunolabeling of the strain ATCC53524 by the antibody against

289 CesA protein, merged on the phase-contrast image. Pretreatment of the cell prior to the primary

290 antibody treatment was as follows: (A) lysozyme treatment followed by detergent treatment (the

291 optimized condition in this study), (B) no pretreatment, (C) EDTA treatment, (D) detergent

292 treatment, (E) EDTA treatment followed by detergent treatment, (F) lysozyme treatment. The inset

293 shows the image at a higher magnification. 

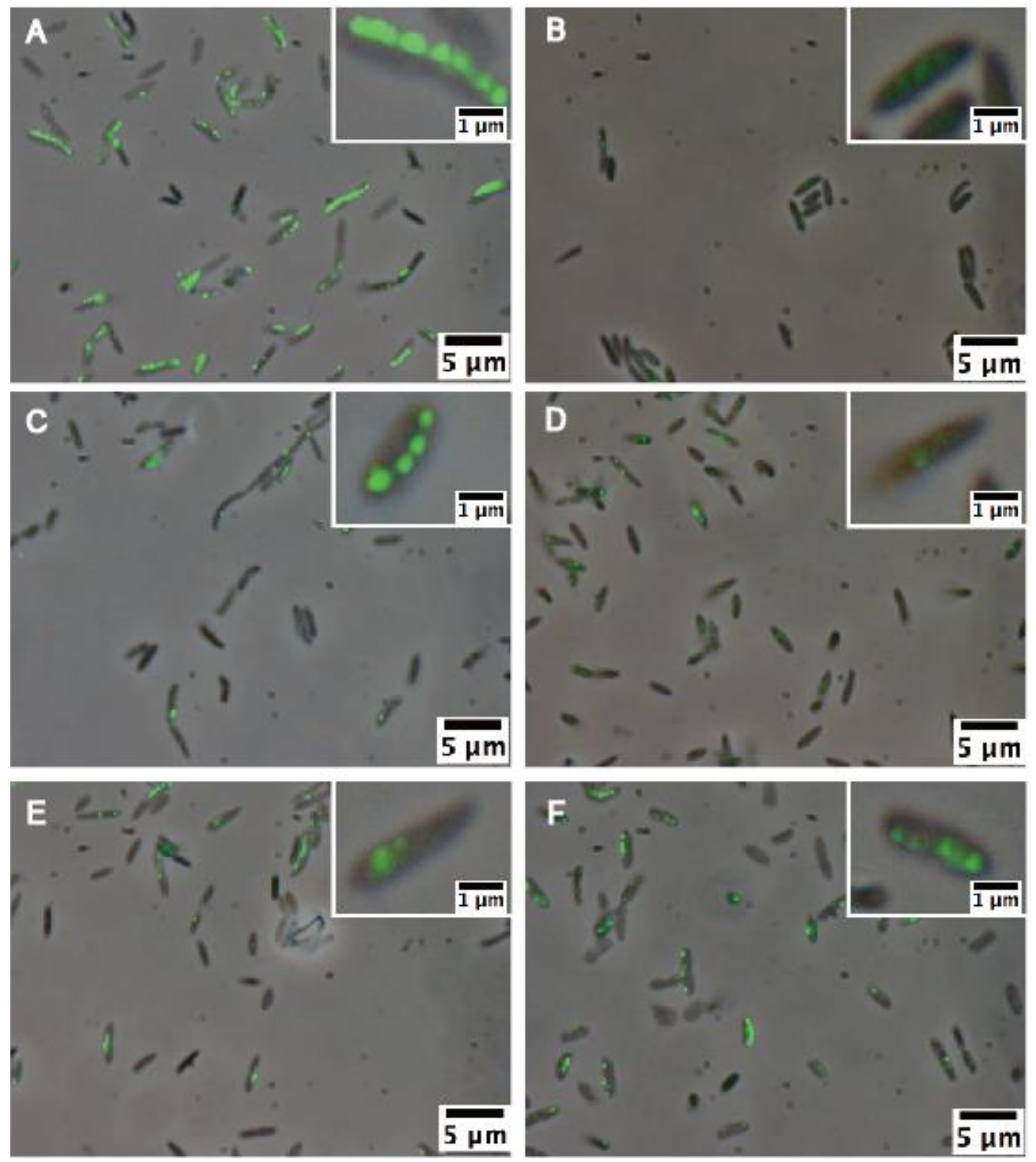

297 Figure 4.

298 Fluorescence micrographs with immunolabeling of the strain ATCC53524 by the antibody against

299 CesD protein, merged on the phase-contrast image. Pretreatment of the cell prior to the primary

300 antibody treatment was as follows: (A) lysozyme treatment followed by detergent treatment (the

301 optimized condition in this study), (B) no pretreatment, (C) EDTA treatment, (D) detergent

302 treatment, (E) EDTA treatment followed by detergent treatment, (F) lysozyme treatment. The inset

303 shows the image at a higher magnification. 


\section{Pre-treatment after PFA fixation \\ CesA \\ CesD}

Lysozyme treatment + Detergent treatment

$+++\quad+++$

No treatment

$\mathrm{TE}^{*}$ treatment

Detergent treatment

TE treatment + Detergent treatment

$++\quad++$

Lysozyme treatment

$++\quad++$

$309-$-: Almost no labeling was found

310 +: A small number of the cells were labeled

$311++$ : A substantial number of the cells were labeled

$312+++$ : Most of the cells were labeled

$313 *$ : Tris-EDTA buffer (100 mM Tris-HCl (pH 6.7), 5 mM EDTA), the same buffer used for the

314 lysozyme treatment

Detergent treatment alone did not allow for the immunolabeling of CesA

317 and CesD, in contrast to the expectation (Figures 3D and 4D). However, EDTA

318 treatment prior to detergent treatment dramatically improved the immunolabeling

319 efficiency for both CesA and CesD (Figures 3E and 4E). This indicates that the

320 permeabilization by the detergent is not sufficient for disturbing the outer

321 membrane of Acetobacter to introduce the antibody to the inside of the cell

322 (periplasm and cytoplasm).

323 A substantial number of cells were immunolabeled when treated with

324 lysozyme alone for both CesA and CesD (Figures 3F and 4F). Given that CesD is 
325 localized in the periplasm, as shown above, the lysozyme treatment without

326 permeabilizing the inner membrane was sufficient to immunolabel CesD protein.

327 However the substantial immunolabeling of CesA protein from such pretreatment

328 requires a speculative interpretation given that the carboxyl terminal of CesA

329 protein (the epitope of the antibody used in this study) is on the cytoplasmic side

330 and prevents access of the antibody unless the inner membrane is permeabilized.

331 We consider that this observation reflects weak but nevertheless significant cell

332 lysis due to the lysozyme treatment.

334 DisCUSSION

This study provides evidence that CesA is included in the TC of bacterial

336 cells, which had already been reported for plant cells (Kimura et al. 1999). Based

337 on fluorescence immuno-microscopy, this study also showed that CesD is

338 included in the linear TC, as reported previously (Sunagawa et al. 2013). These

339 observations are not sufficient to conclude that the CesA and CesD proteins are

340 colocalized in the TC. Direct immunolabeling with a fluorescence dye-labeled

341 primary antibody should provide a clearer conclusion for the colocalization of

342 CesA and CesD proteins in the TC. Neverthelss, the linear labeling pattern

343 observed for CesA and CesD in this study is striking enough to propose that CesA

344 and CesD are colocalized in the TC of Acetobacter, regardless of whether their

345 interaction is direct or indirect.

346 The structural models for the CesA/CesB complex (Morgan et al. 2016,

347 Morgan et al. 2013, Morgan et al. 2014) and CesD (Hu et al. 2010) also support

348 the functional link between CesA and CesD, given that the former generates

349 cellulose from UDP-glucose and the latter includes cellulose chains in the channel 
350 formed by its homo-octamer. It is then proposed that CesD functions downstream

351 of the CesA/CesB complex in the process of cellulose biosynthesis, and that they

352 are spatially close. This hypothesis is consistent with the observation that

353 immunolabeling of CesA and CesD proteins showed a linear pattern in the cells in

354 this study.

355 We also attempted the immunolabeling of CesB and CesC protein in this

356 study although no successful data were obtained. For CesC protein, which is

357 currently the most enigmatic subunit, the reason for the failure is unclear. A

358 possible reason could be related to access of the antibody to the epitope, which is

359 significantly influenced by the stereo arrangement of this subunit in the cell.

360 However, it was unexpected that the immunolabeling of CesB protein did not

361 show a linear labeling pattern as previously observed by SDS-FRL (Kimura et al.

362 2001). It is noticeable that the antibody against CesB used in this study allowed

363 for the linear TC to be labeled by SDS-FRL (Figure 5), despite a relatively high

364 non-specific labeling probably owing to that it is a polyclonal antibody. This

365 result indicates that this antibody is able to label CesB protein in the SDS-treated

366 freeze-replica prepared from the cells fixed by flash-freezing with no chemicals.

367 Therefore, a possible interpretation for this unexpected result is that the PFA

368 fixation might kill the epitope activity of the CesB protein, for example by

369 changing the protein itself and/or its surrounding environment, so as to inhibit

370 binding of the antibody in immunofluorescence labeling. 


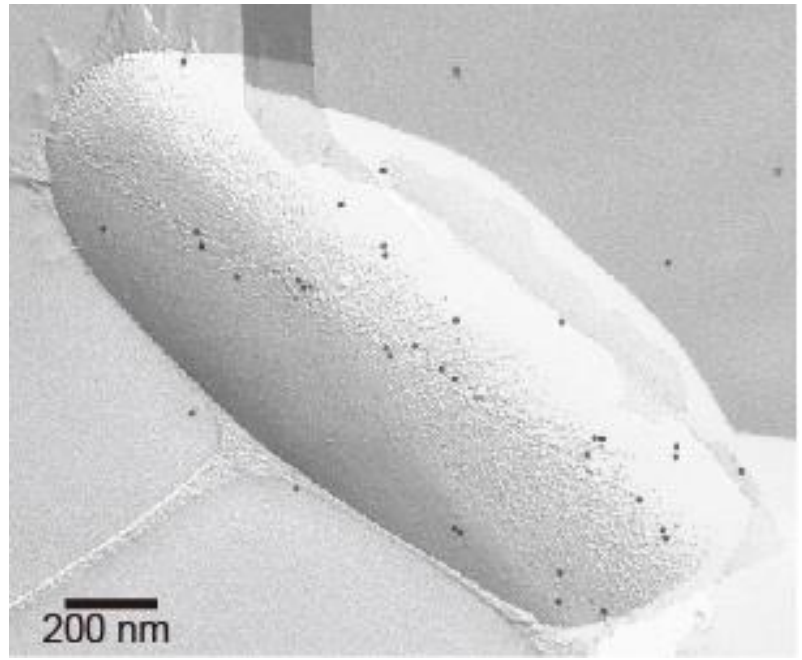

375 Figure 5.

376 Electron micrograph of SDS-FRL for the strain ATCC53524 with the antibody against CesB

377 protein. The antibody location was visualized by colloidal gold of a $15 \mathrm{~nm}$ diameter. Linear pattern

378 of the labeling was clearly found despite a relatively high non-specific antibody labeling, which is

379 probably due to that the antibody is a polyclonal antibody against peptide.

The antibody against CesA protein, which gave successful

382 immunolabeling as shown in Figure 2A, was also used for SDS-FRL to visualize

383 CesA protein in Acetobacter TC. However to date, no labeling for CesA protein

384 was found on the replica despite successful labeling for CesB protein as shown

385 above. This is probably due to that CesA protein in the inner membrane was

386 detached from replica of the outer membrane by solubilization with SDS.

387 Significant improvement will be required for successful SDS-FRL with the 388 antibody against CesA protein.

389 Compiling the results of this and previous studies, we propose a

390 hypothetical model for the TC of Acetobacter as shown in Figure 6. The

$391 \mathrm{CesA} / \mathrm{CesB}$ complex is embedded in the inner membrane, given that the ligands

392 (UDP-glucose and c-di-GMP) are cytosolic molecules and the product cellulose is

393 extruded outside through the membrane-spanning channel (Morgan et al. 2013). 
394 CesC is depicted as the cellulose-translocating channel in the outer membrane

395 according to the currently accepted model (McNamara et al. 2015, Saxena et al.

396 1994). No immunolabeling from the lack of pretreatment, and weak

397 immunolabeling from EDTA treatment alone for CesD protein indicated that

398 CesD is located in the periplasmic space. The result of a biochemical study using

399 marker enzyme assays also support this hypothesis (Iyer et al. 2011). Given that

400 the function of CesD is carried out downstream of CesA as discussed above, CesD

401 protein is located close to the exit of the cellulose-translocation channel of CesA

402 protein in the periplasm, as proposed based on a previous structural analysis of the

403 Acetobacter CesA/CesB complex with electron microscopy (Du et al. 2016).

$404 \quad$ Given that one CesA/CesB complex produces one cellulose chain

405 (Morgan et al. 2013), and the CesD oligomer includes four chains in its inner pore

406 (Hu et al. 2010), the model in Figure 6a represents only one CesA/CesB complex,

407 and the other three complexes are not shown for visual clarity. A combination of

408 these molecules could be the functional unit to produce the primary assembly of

409 the polymerized cellulose chains prior to microfibril formation, which has been

410 proposed as a "mini-sheet" in a previous study (Cousins and Brown Jr. 1995). The

411 linear array of this whole complex should be visualized as the linear type TC in

412 Acetobacter (Figure 6b). 


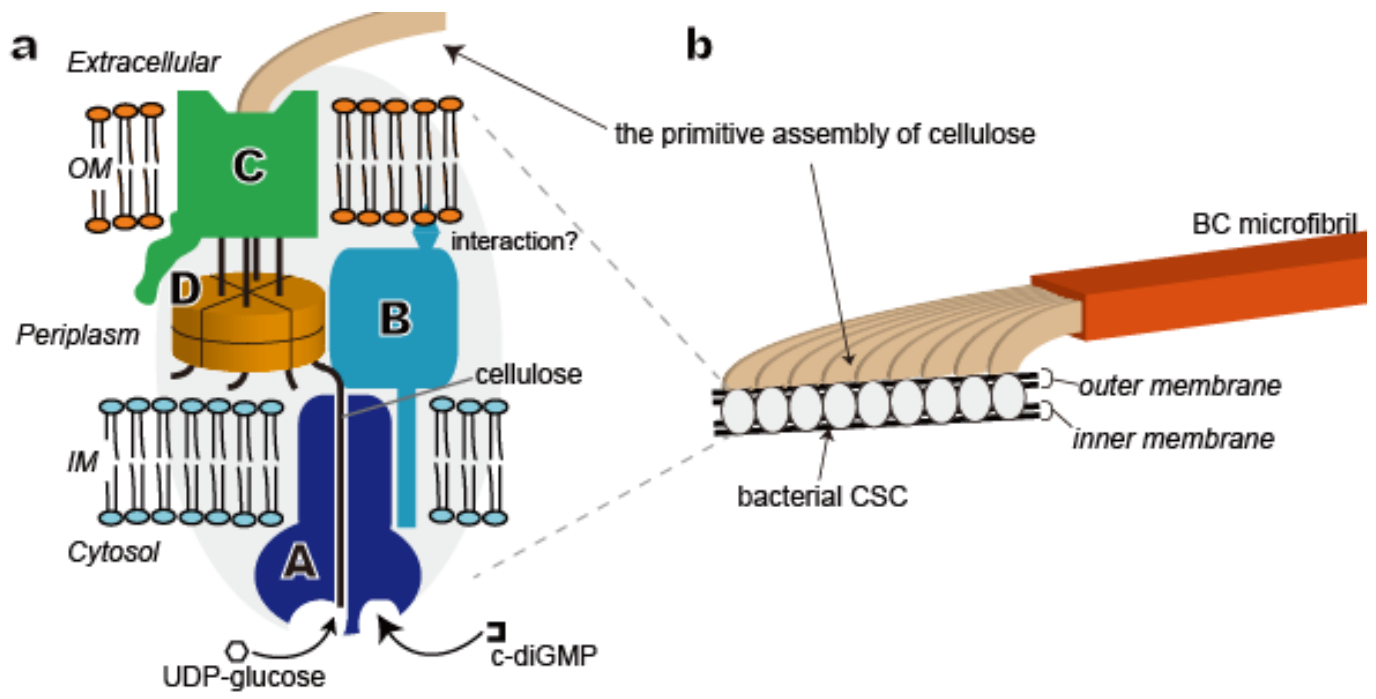

$414 \quad$ Figure 6.

415 A schematic model for the cellulose synthase complex of Acetobacter. In the schematic diagram of 416 the subunit location in one complex (a), CesA and CesB are depicted as monomers while CesD is

417 illustrated as an octamer through which four cellulose chains pass, as reported previously (Hu et al.

418 2010). CesC is located in the outer membrane according to the currently accepted model

419 (McNamara et al. 2015, Saxena et al. 1994). The terminal complexes are probably formed by the

420 linear array of these complexes as shown in (b). OM: outer membrane; IM: inner membrane

The SDS-FRL experiment also provided insight about CesB protein.

423 Given the smoothness of the fractured surface, the linear TC of Acetobacter is

424 found in the P-face (the extracellular surface of the inner leaflet of the lipid

425 bilayer) of the outer membrane (Kimura et al. 2001). The successful

426 immunolabeling of CesB protein by SDS-FRL indicates that this protein remains

427 with the replica even after SDS-treatment, indicating that CesB protein

428 significantly interacts with the outer membrane from the periplasmic side. This

429 interaction is likely important for guiding the cellulose chain to the extracellular

430 side and/or the crystallization of cellulose chains into a microfibril. Further SDS-

431 FRL experiment with other antibodies will shed light on the locations of the other

432 subunits. 


\section{CONCLUDING REMARKS}

435 This study demonstrated that CesA, the core catalytic subunit of cellulose

436 synthase, is the molecule included in the linear-type TC or the CSC of

437 Acetobacter. Structural analysis of these proteins has recently started providing

438 many insights about the enzymatic mechanism of cellulose synthase as well as

439 other well-known membrane proteins such as ion/water channels and transporters.

440 However, for cellulose synthase, which functions in the assembly of polymer

441 chains into a supermolecular aggregation, the structural analysis of the protein

442 complex at a cellular/subcellular scale is important for understanding the

443 underlying mechanism. Further studies with microscopy will play an important

444 role for shedding light on the mechanism of cellulose chains assembly into the

445 microfibril.

447 Acknowledgements

448 The authors declare no conflicts of interest. This study was supported by CREST, JST to JS,

449 KAKENHI [25650033; 15H04530] from the Japan Society for the Promotion of Science (JSPS) to

450 TI, and the Humanosphere Mission Research (2009, RISH, Kyoto University) to TI and SK.

451 Electron microscopic observations were performed by the Analysis and Development System for

452 Advanced Materials (ADAM), in RISH, Kyoto University. We would like to thank Editage

453 (www.editage.jp) for English language editing. 
References

Cousins SK, Brown Jr. RM (1995) Cellulose I microfibril assembly: computational molecular mechanics energy analysis favours bonding by van der Waals forces as the initial step in crystallization. Polymer 36:3885-3888 Du J, Vepachedu V, Cho SH, Kumar M, Nixon BT (2016) Structure of the cellulose synthase complex of Gluconacetobacter hansenii at 23.4 Å resolution. PLoS ONE 11: 3e0155886. doi: 10.1371/journal.pone.0155886

463 Fujimoto K (1995) Freeze-fracture replica electron microscopy combined with SDS digestion for

464 cytochemical labeling of integral membrane proteins - Application to the immunogold labeling of

465 intercellular junctional complexes. J Cell Sci 108:3443-3449

466 Hashimoto A, Shimono K, Horikawa Y, Ichikawa T, Wada M, Imai T, Sugiyama J (2011)

467 Extraction of cellulose-synthesizing activity of Gluconacetobacter xylinus by alkylmaltoside.

468 Carbohydr Res 346:2760-2768

469 Hu S-Q, Gao Y-G, Tajima K, Sunagawa N, Zhou Y, Kawano S, Fujiwara T, Yoda T, Shimura D,

470 Satoh Y, Munekata M, Tanaka I, Yao M (2010) Structure of bacterial cellulose synthase subunit D 471 octamer with four inner passageways. Proc Natl Acad Sci U S A 107:17957-17961

472 Imai T, Sun S-j, Horikawa Y, Wada M, Sugiyama J (2014) Functional reconstitution of cellulose

473 synthase in Escherichia coli. Biomacromolecules 15:4206-4213

474 Itoh T, Kimura S, Brown Jr. RM (2007) Immunogold labeling of cellulose-synthesizing terminal

475 complexes. In: Brown Jr. RM, Saxena IM (ed) Cellulose: Molecular and Structural Biology.

476 Springer: Netherlands, pp237-255.

477 Iyer PR, Catchmark J, Brown NR, Tien M (2011) Biochemical localization of a protein involved

478 in synthesis of Gluconacetobacter hansenii cellulose. Cellulose 18:739-747

479 Kawano S, Tajima K, Kono H, Numata Y, Yamashita H, Satoh Y, Munekata M (2008) Regulation

480 of endoglucanase gene (cmcax) expression in Acetobacter xylinum. J. Biosci. Bioengin. 106:88-94

481 Kawano S, Tajima K, Kono H, Erata T, Munekata M, Takai M (2002) Effects of endogenous

482 endo- $\beta-1,4$-glucanase on cellulose biosynthesis in Acetobacter xylinum ATCC23769. J. Biosci.

483 Bioengin. 94:275-281

484 Kimura S, Chen HP, Saxena IM, Brown R.M. J, Itoh T (2001) Localization of c-di-GMP-binding

485 protein with the linear terminal complexes of Acetobacter xylinum. J Bacteriol 183:5668-5674

486 Kimura S, Laosinchai W, Itoh T, Cui X, Linder CR, Brown Jr. MR (1999) Immunogold labeling

487 of rosette terminal cellulose-synthesizing complexes in the vascular plant Vigna angularis. Plant

488 Cell 11:2075-2085

489 McNamara JT, Morgan JLW, Zimmer J (2015) A molecular description of cellulose biosynthesis.

490 Ann. Rev. Biochem. 84:895-921

491 Morgan JL, McNamara JT, Fischer M, Rich J, Chen HM, Withers SG, Zimmer J (2016) Observing

492 cellulose biosynthesis and membrane translocation in crystallo. Nature 531:329-334

493 Morgan JLW, McNamara JT, Zimmer J (2014) Mechanism of activation of bacterial cellulose

494 synthase by cyclic di-GMP. Nat. Struct. Mol. Biol. 21:489-496 
495 Morgan JLW, Strumillo J, Zimmer J (2013) Crystallographic snapshot of cellulose synthesis and

496 membrane translocation. Nature 493:181-186

497 Nakai T, Sugano Y, Shoda M, Sakakibara H, Oiwa K, Tuzi S, Imai T, Sugiyama J, Takeuchi M,

498 Yamauchi D, Mineyukia Y (2013) Formation of highly twisted ribbons in a

499 carboxymethylcellulase gene-disrupted strain of a cellulose-producing bacterium. J Bacteriol

$500 \quad 195: 958-964$

501 Nakai T, Moriya A, Tonouchi N, Tsuchida T, Yoshinaga F, Horinouchi S, Sone Y, Mori H, Sakai

502 F, Hayashi T (1998) Control of expression by the cellulose synthase (bcsA) promoter region from

503 Acetobacter xylinum BPR 2001. Gene 213:93-100

504 Omadjela O, Narahari A, Strumillo J, Mélida H, Mazur O, Bulone V, Zimmer J (2013) BcsA and

505 BcsB form the catalytically active core of bacterial cellulose synthase sufficient for in vitro

506 cellulose synthesis. Proc Natl Acad Sci U S A 110:17856-17861

507 Saxena IM, Kudlicka K, Okuda K, Brown Jr. RM (1994) Characterization of genes in the

508 cellulose-synthesizing operon (acs operon) of Acetobacter xylinum: Implications for cellulose

509 crystallization. J Bacteriol 176:5735-5752

510 Schramm M, Hestrin S (1954) Factors affecting production of cellulose at the air/liquid interface

511 of a culture of Acetobacter xylinum. J Gen Microbiol 11:123-129

512 Somerville C (2006) Cellulose synthesis in higher plants. Ann. Rev. Cell Dev. Biol. 22:53-78

513 Standal R, Iversen T-G, Coucheron DH, Fjaervik E, Blatny JM, Valla S (1994) A new gene

514 required for cellulose production and a gene encoding cellulolytic activity in Acetobacter xylinum

515 are colocalized with the bcs operon. J Bacteriol 176:665-672

516 Sun S-j, Horikawa Y, Wada M, Sugiyama J, Imai T (2016) Site-directed mutagenesis of bacterial

517 cellulose synthase highlights sulfur-arene interaction as key to catalysis. Carbohydr Res 434:99-

518 106. doi: 10.1016/j.carres.2016.08.009

519 Sunagawa N, Fujiwara T, Yoda T, Kawano S, Satoh Y, Yao M, Tajima K, Dairi T (2013)

520 Cellulose complementing factor (Ccp) is a new member of the cellulose synthase complex

521 (terminal complex) in Acetobacter xylinum. J. Biosci. Bioengin. 115:607-612

522 Wong HC, Fear AL, Calhoon RD, Eichinger GH, Mayer R, Amikam D, Benziman M, Gelfand

523 DH, Meade JH, Emerick AW, Bruner R, Ben-Bassat A, Tal R (1990) Genetic organization of the

524 cellulose synthase operon in Acetobacter xylinum. Proc Natl Acad Sci U S A 87:8130-8134 